\title{
Neuromapping of the Capsuloligamentous Knee Joint Structures
}

\author{
Andreas Martin Seitz, Ph.D., Miriam Murrmann, M.D., Anita Ignatius, Ph.D., \\ Lutz Dürselen, Ph.D., Benedikt Friemert, M.D., and Falk von Lübken, M.D.
}

\begin{abstract}
Purpose: To investigate neuromuscular electromyographic response of the of the upper and lower leg muscles after the application of an intraoperative, isolated mechanical stimulus of the capsuloligamentous structures, including the anterior (ACL) and posterior cruciate ligaments (PCL), lateral (LM) and medial menisci (MM), plica mediopatellaris (PM), and Hoffa's fat pat (HFP). Methods: The electromyographic response of the upper and lower leg muscles (M. rectus femoris; M. vastus medialis; M. semitendinosus; M. biceps femoris; M. gastrocnemius lateralis) of 15 male patients were measured after an isolated mechanical stimulus of the capsuloligamentous structures during an arthroscopic intervention using a customized intraoperative setup. Target parameters were the short (SLR; $<30$ milliseconds) and medium latency responses (MLR; >30 milliseconds) after the mechanically-induced trigger. Results: The ACL, PCL, LM, and MM displayed high interindividual reproducibility of $>76 \%$. The MM was the only structure indicating both an SLR and MLR for all muscles. Although signals could be detected, there was no reproducibility in electromyographic signal activation for the HFP. The most rapid MLR was observed for the PM (quadriceps: 37 milliseconds). Conclusions: Each stimulated structure displayed an individual MLR response, which allowed us to create neuromapping combining the anatomical and quantitative representations of the individual muscular activation patterns after isolated mechanical stimulation of the capsuloligamentous knee joint structures, corroborating our hypothesis. Level of Evidence: Diagnostic - Level II.
\end{abstract}

$\mathbf{W}$ ith an incidence of $37 \%$, the knee is one of the most frequently injured joints during sports activities. ${ }^{1}$ Injuries of the capsuloligamentous structures of the knee are one of the most common diagnoses in everyday clinical practice, showing a high incidence both after sports injuries and trauma and in the context

From the Institute of Orthopedic Research and Biomechanics; Institute of Orthopedic Research and Biomechanics; Center for Trauma Research Ulm, Ulm University Medical Center (A.M.S., M.M., A.I., L.D.); and Department of Trauma and Orthopedic Surgery, Hospital of the Federal Armed Forces Ulm (B.F., F.v.L.), Ulm, Germany.

The authors report that they have no conflicts of interest in the authorship and publication of this article. Full ICMJE author disclosure forms are available for this article online, as supplementary material.

Part of this work was presented at the 2019 annual meeting of the Orthopaedic Research Society (ORS) in Austin, Texas, U.S.A., and awarded with the prestigious NIRA award.

Received July 14, 2020; accepted December 29, 2020.

Address correspondence to Andreas Martin Seitz, Ph.D., Institute of Orthopedic Research and Biomechanics, Center for Trauma Research Ulm, Ulm University Medical Center, Helmholtzstr. 14; 89081 Ulm, Germany. E-mail: andreas.seitz@uni-ulm.de

(C) 2021 THE AUTHORS. Published by Elsevier Inc. on behalf of the Arthroscopy Association of North America. This is an open access article under the CC BY license (http://creativecommons.org/licenses/by/4.0/).

2666-061X/201231

https://doi.org/10.1016/j.asmr.2020.12.009 of degenerative diseases. ${ }^{1,2}$ The knee joint can be injured by a direct impact in contact sports, including football and soccer, or by a rotational movement without the influence of an external opponent, for example, during skiing activities. The directly associated treatment costs are themselves of socioeconomic importance, and the majority of patients affected are in the workforce. ${ }^{3}$ The associated indirect follow-up costs caused by loss of working hours and early arthrosis are difficult to quantify, but they are assumed to be at least 4 times greater than the direct costs. ${ }^{3}$

Flexion-valgus external rotation trauma of the knee joint is one of the most frequent noncontact injury mechanisms $^{2,4}$ and can lead to a severe combination trauma of the capsuloligamentous knee joint structures, which is commonly associated with a loss of proprioception and knee joint stability. The resulting loss of proprioception and instability can be caused mechanically as well as neuromuscularly. The latter is also known as a functional instability and can be explained by a disturbance of the sensorimotor regulation of the knee joint. The hereby damaged mechanoreceptors lose their function to adequately provide an afferent stimulus. Loss of this reflex leads, by a loss of muscle coordination, to a loss of protective joint function. The 
clinical equivalent to this are giving-way episodes, resulting in a continuously increasing instability symptomatology, ${ }^{5}$ which can lead in the long term to micro-and macrocartilage lesions and finally to premature osteoarthritis of the knee.

In addition to their function as mechanical stabilizers, the anterior (ACL) and posterior (PCL) cruciate ligaments play an important role within the neuromuscular feedback mechanism. ${ }^{6-14}$ Clinically, there are 2 different types of patients presenting with ACL concerns: coper and noncopers. In contrast to the patients who are copers, that is, they feel subjectively stable and are able to largely resume pretraumatic activity, patients who are noncopers exhibit considerable subjective functional instability without any indication of a severe mechanical instability, leading to the so-called giving-way symptom (GWS). ${ }^{15-17}$ Chmielewski et al. ${ }^{15}$ described functional joint stability as the ability of the knee joint to remain stable during everyday physical movements without displaying a GWS. The GWS can be clinically defined as a disturbed reflex control loop, creating an undesired muscular relaxation around the knee, which leads to an insufficient stabilization and thus to a subluxation of the knee. In human and animal experiments, a reflex control loop was identified between the ACL and the hamstring muscles. ${ }^{8,9,13,14,18-21}$ It is established that the different neuroreceptors in the tissues of the knee joint ${ }^{22,23}$ contribute to a different extent to the proprioceptive function of the healthy and degenerated joint. $^{24,25}$ Therefore, it is possible that other capsular and ligamentous structures also contribute to the functional stability of the knee by similar reflex control loops. ${ }^{26,27}$

Because of the severity of the injury, the limited therapeutic options, and the according risk factors that are very important for the long-term outcome, the adequate treatment of capsuloligamentous injuries of the knee joint is of paramount importance. The primary aim of this study was to test whether knee joint structures other than the ACL and PCL contribute to knee joint proprioception. One of the major issues of recent detection methods is that when an external mechanical stimulus is triggered, multiple receptors of the knee joint can be activated, resulting in a specific neuromuscular activation. However, it is unknown which single anatomical structure causes the observed muscular response. ${ }^{28,29}$ The purpose of this study was to investigate neuromuscular electromyographic response of the of the upper and lower leg muscles after the application of an intraoperative, isolated mechanical stimulus of the capsuloligamentous structures, including the ACL, PCL, lateral meniscus (LM), medial meniscus (MM), plica mediopatellaris (PM), and Hoffa's fat pad (HFP). We hypothesized that an isolated mechanical stimulus not only of the ACL or PCL but also of the menisci, PM, or the HFP creates a distinct neuromuscular electromyographic (EMG) response of the upper and lower leg muscles.

\section{Methods}

\section{Patients}

Following institutional review board approval (Ulm University, no. 91/11) and the patients' written informed consent, the ACL, PCL, LM and MM, PM, and HFP of 17 male patients (Table 1) with no history of neurologic disorders were investigated during different arthroscopic interventions. Because of interference frequencies of the EMG signals in 1 patient (patient 1) and poor recording of the mechanical trigger signal in another patient (patient 7), we were able to evaluate measurements from 15 patients. All patients underwent general anesthesia (total intravenous anesthesia) for the surgical procedure with 50 to $150 \mu \mathrm{g} / \mathrm{kg} / \mathrm{min}$ propofol. The use of such low doses is unlikely to affect the monosynaptic reflex arc. ${ }^{30}$ No further relaxants or local anesthetics, spinal anesthesia, or catheters were used before or during the measurements.

\section{Measurement Setup}

A novel customized muscle reflex measurement setup (Fig 1) was developed to allow for an isolated mechanical stimulation of the capsuloligamentous structures. Hereby, a commercially available arthroscopic hook probe (Richard Wolf GmbH, Knittlingen, Germany) with a modified rounded tip that was directly connected to an impactor allowed for the application of isolated subcritical stimuli of $15-\mathrm{N}$ tensile force to the PM and HCP and $40 \mathrm{~N}$ to the ACL, PCL, LM, and MM (Fig 2). The impactor's force signal was amplified and connected to an input box (USB Input box; Biovision, Wehrheim, Germany). The input box, which was connected via USB to the measurement computer, was also able to handle and process the EMG. The raw neuromuscular EMG data of the thigh and shank muscles (M. vastus medialis, $M$. rectus femoris, $M$. semitendinosus, $\mathrm{M}$. biceps femoris, $\mathrm{M}$. gastrocnemius lateralis) were collected at a sampling rate of $3 \mathrm{kHz}$, $1000 \times$ preamplified, and recorded with a commercially available evaluation software (Daisy Lab; Biovision). Electrode placement was conducted in accordance to the SENIAM guidelines ${ }^{31}$ using 2 adhesive electrodes (Kendall; Covidien, Dublin, Ireland) for each muscle head, while the reference electrode was positioned at the iliac spine. To achieve an optimal electrode contact with low skin impedance, the skin was shaved and cleaned with alcohol. The EMG signals were band-pass filtered (10-500 Hz) with an eighth-order Butterworth filter to eliminate artifacts. Validation and repeatability of linear envelope EMG measurements were ensured during pretests. 
Table 1. Demographic Patient Data

\begin{tabular}{|c|c|c|c|c|}
\hline No. & Age, y & Height, cm & Weight, kg & Indication \\
\hline $1^{*}$ & 19 & 183 & 72 & ARY, microfracture \\
\hline 2 & 20 & 185 & 95 & Diagnostic ARY \\
\hline 3 & 26 & 176 & 76 & ARY, MPFL Recon \\
\hline 5 & 21 & 193 & 120 & ARY, ACL Recon: BTB \\
\hline 6 & 34 & 186 & 95 & ARY, ACL Recon: BTB \\
\hline $7^{\#}$ & 44 & 193 & 82 & Diagnostic ARY \\
\hline 10 & 48 & 175 & 88 & ARY, ACL Recon: STG \\
\hline 11 & 33 & 186 & 92 & ARY, HTO \\
\hline 12 & 21 & 169 & 73 & ARY, ACL Recon: STG \\
\hline 13 & 22 & 190 & 90 & ARY, Drill hole filling \\
\hline 14 & 31 & 189 & 103 & ARY, ACL Recon: STG \\
\hline 15 & 23 & 178 & 72 & Diagnostic ARY \\
\hline
\end{tabular}

NOTE. The last row summarizes the mean \pm standard deviation values.

ACL, anterior cruciate ligament; ARY, arthroscopy; BTB, bone to bone graft; HTO, high tibial osteotomy; MPFL, medial patellofemoral ligament; Recon, reconstruction; STG, semitendinosus graft.

*Patients were excluded due to technical issues.

\section{In Vitro: Tensile Test}

Pretests were conducted to prevent any injury to the capsuloligamentous structures during the in vivo measurements. Therefore, 6 intact cadaveric porcine stifle joints, obtained from a local butcher, were tested using a customized setup in a standard material testing machine (Fig 3; Z010; Zwick GmbH, Ulm, Germany). The porcine joints were potted in steel cylinders using polymethylmethacrylate (Technovit 3040; Kulzer GmbH, Hanau, Germany) and mounted in a tibial jig, which allowed free placement in 5 degrees of freedom. Subsequently, an arthroscopic hook, which was connected to a $1-\mathrm{kN}$ load cell, applied maximum tensile loads of 50 $\mathrm{N}$ to the HFP and PM and $500 \mathrm{~N}$ to all other capsuloligamentous structures at a loading rate of 100 $\mathrm{mm} / \mathrm{min}$. During the measurements, the force elongation curves were recorded and the rupture force and the force at the transition from the toe to the linear region (Table 2), reflecting the typical behavior of soft tissues, $^{32}$ were determined. This transition point was defined as the threshold for subcritical loads. Subsequently, we defined subcritical loads far below the failure loads of $40 \mathrm{~N}$ for the ACL, PCL, LM and MM and of $15 \mathrm{~N}$ for the HFP and PM, which were then used during the intraoperative mechanical stimulation.

\section{In Vivo Measurements}

The patients were positioned for arthroscopic knee surgery on the operating table and subsequently all electrodes were placed as described above and covered with sterile tape. A tourniquet (Tourniquet 5000; VBM Medizintechnik, Sulz am Neckar, Germany) was placed around the thigh and inflated for the surgical procedures after the measurements. Once the leg was cleaned and draped, a diagnostic arthroscopy was performed using an anterolateral approach. The sterile hook was inserted via the anteromedial approach. The stimuli of the ACL, PCL, and MM were conducted with the hook directly attached. For the stimulation of the LM, the approaches were changed, with insertion of the optics to the medial portal and the hook to the anterolateral portal. By contrast, the stimuli of the PM and HFP were performed using a surgical clamp that provided a larger application surface to which the hook was directly interlocked. The intraoperative measurements were repeated three times, while the tibia was fixed by means of fixation tape to prevent anterior tibial displacement. Following completion of the measurements, the indicated surgical procedure was performed. The target value of the measurements was the latency time between the isolated mechanical stimulation of the capsuloligamentous structures and the increase of the EMG potential of the knee-spanning muscles. Latencies $<30$ milliseconds are defined as short latency responses (SLR, monosynaptic tendon jerk reflexes), whereas latencies $\geq 30$ milliseconds are defined as medium latency responses (MLR, polysynaptic spinal reflexes). ${ }^{28}$ A previously described algorithm was used to differentiate between the end of the SLR and the beginning of the MLR. ${ }^{28}$

\section{Statistical Analysis}

Following a priori sample calculation $\left(\mathrm{G}^{*}\right.$ Power $3.1^{33}$ : $\alpha=0.05, \beta=0.2, d z=2.08 ; n=5)$, the mean latency response (SLR, MLR) of each capsuloligamentous structure was evaluated. 


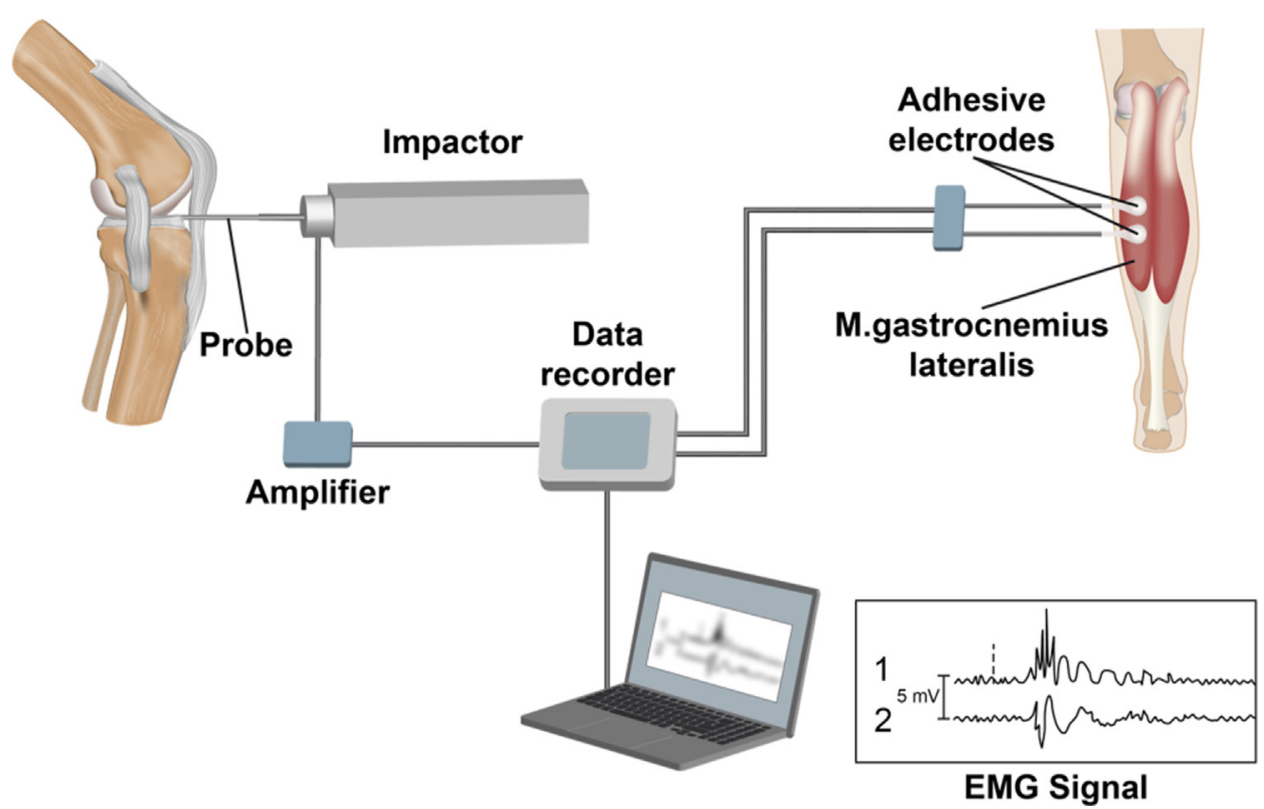

Fig 1. Schematic drawing of the test setup to measure the latency time between the isolated mechanical stimulation of the capsuloligamentous structure via the impactor and the increase of the electromyographic potential of the knee-spanning muscles (here: M. gastrocnemius).

\section{Results}

The mechanical stimulation of the ACL led to reproducible $(\mathrm{n}=15 ; 98.3 \%)$ muscle response characteristics of all 5 muscles, which indicated an MLR ranging between 43 and 79 milliseconds (Fig 4). Regarding the SLR, only the M. vastus medialis displayed a reproducible response between 9 and 15 milliseconds.

With respect to the quadriceps and hamstring muscles, the isolated ACL and PCL stimulation caused a contrary reflex response. Following PCL stimulation $(\mathrm{n}=12 ; 100 \%$ MLR reproducibility), the primary reflex response of the hamstrings occurred after 40 to 50 milliseconds with a subsequent quadriceps response. For the ACL stimulation the MLR response was vice versa.

Both for the LM and the MM the first MLR was detected for the M. rectus femoris (40-60 milliseconds) followed by the M. biceps femoris. Overall, both menisci displayed qualitatively similar EMG responses, except for the M. gastrocnemius lateralis. In contrast to the hamstring muscles, the quadriceps muscles indicated an SLR after the isolated stimulation of the LM (range 10-15 milliseconds). Differences were detected in the activation profile of the M. rectus femoris $(\mathrm{n}=21 ; 94.6 \%$ reproducibility), whereas all other muscles indicated a high interindividual reproducibility. The MM was the only capsuloligamentous structure that indicated both an SLR (range: 10-24 milliseconds) and an MLR (range: 33-83 milliseconds) for all 5 muscles with a good reproducibility $(\mathrm{n}=24 ; 76.2 \%)$.

Although some signals could be detected for the HFP, there was no reproducibility in the EMG signal $(\mathrm{n}=18$; $14.6 \%$ ). The PM displayed the most rapid MLR at the quadriceps muscles (37 milliseconds) of all investigated

structures. The response of the hamstring muscles was 30 to 40 milliseconds later. In general, the reproducibility of the muscle activation was low $(\mathrm{n}=18$; $30.6 \%)$.

The neuromapping (Fig 5) combined the anatomical and quantitative representation of the individual muscular activation patterns after isolated mechanical stimulation of the ACL, PCL, LM, MM, HFP, and PM. The mean MLR was identified as the polysynaptic spinal reflex response and analyzed over time to establish the neuromapping. For the ACL, PCL, LM, and MM, a

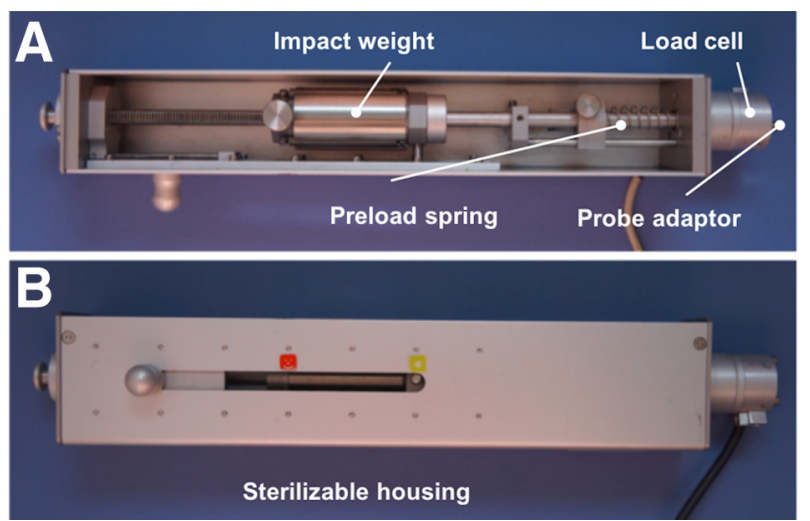

Fig 2. (A) Impactor allowing for an isolated mechanical stimulation of the capsuloligamentous knee joint structures (anterior and posterior cruciate ligament, lateral and medial meniscus, joint capsule, Hoffa's fat pad). An arthroscopic hook probe can be directly attached to the load cell (8426-500 N, burster präzisionsmesstechnik $\mathrm{GmbH}$, Germany) using the probe adaptor. (B) A defined force of $15 \mathrm{~N}$ (red position) or 40 $\mathrm{N}$ (yellow position) can be applied by tensioning the preload spring together with the impact weight to the respective position. 


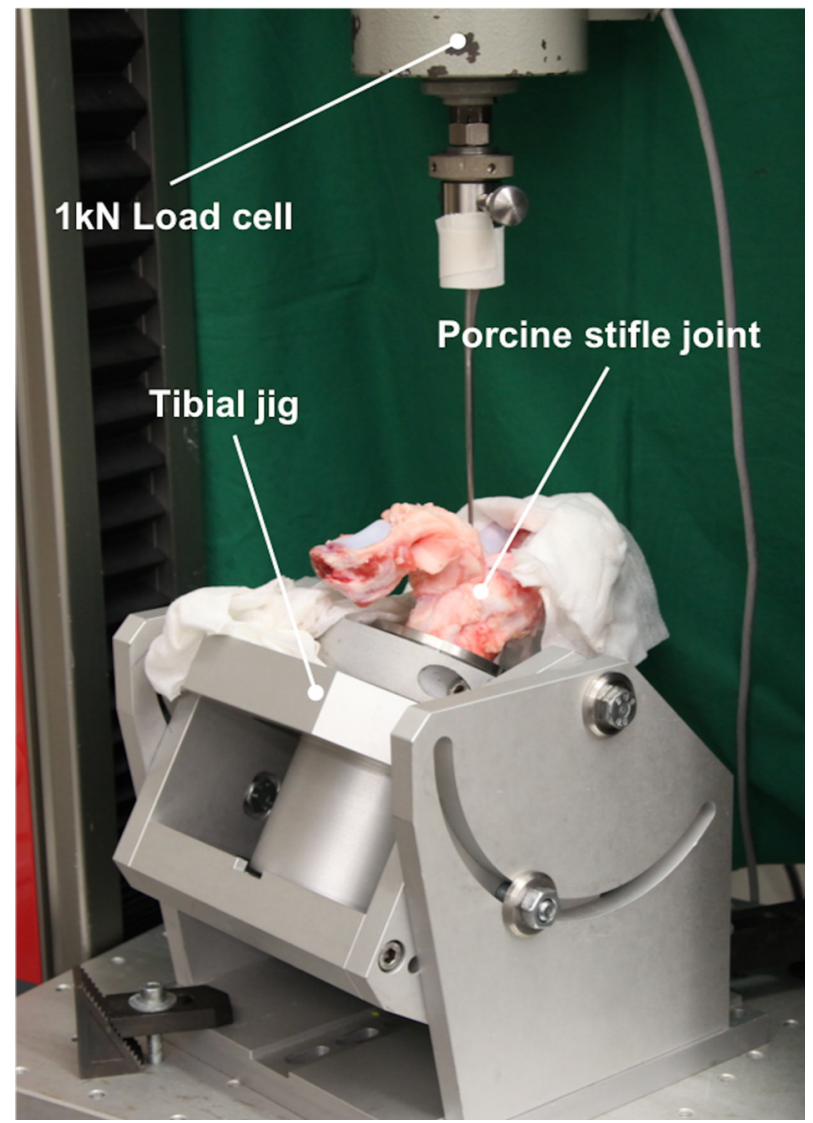

Fig 3. Overview of the tensile test setup with the porcine stifle joint aligned for testing of the lateral meniscus.

reproducible MLR was determined for all 5 muscles. Following isolated stimulation of the PM, only the M. vastus medialis displayed a reproducible reflex response. Stimulation of the HFP led to no reproducible MLR response.

\section{Discussion}

The isolated intraoperative stimulation of the ACL and PCL, LM and MM, and PM resulted in distinct reproducible neuromuscular EMG responses of the thigh and shank muscles, corroborating our hypothesis. In detail, the direct neuromuscular connections between the PCL, LM, MM, the quadriceps, hamstring, and lateral gastrocnemius muscles were described for the first time. In addition, the PM, as part of the capsule, showed a direct connection to the $\mathrm{m}$. vastus medialis. Although without clear reproducibility, patient-specific, independent muscle reflex responses could also be demonstrated for the HFP. The connection between the ACL and the thigh muscles was reestablished and extended by the direct reflex arc to the hamstring muscles.

\section{Latency Times}

An overview of current literature values of latency times after stimulation of the cruciate ligaments and menisci is given in Table 3. The MLR after isolated ACL stimulation ranged from 43 to 79 milliseconds in the current study. The MLR of the hamstring muscles (48-79 milliseconds) followed those of the quadriceps musculature (43-61 milliseconds). These findings are in good agreement with those of Friemert et al. ${ }^{29}$ In their study, they investigated a similar patient cohort $(\mathrm{n}=10)$ and initiated a mechanically induced neuromuscular response between the ACL and the hamstring muscles. They used an arthroscopic probe to transmit a mechanical stimulus of up to $300 \mathrm{~N}$ by means of a dropweight impact to the ACL. The hamstring MLRs of the present study are slightly greater than those from Friemert et al. (40-50 milliseconds), which can be explained by a more rapid potential change of the hamstrings in the study of Friemert et al. resulting from a greater maximum force of $300 \mathrm{~N}$ compared with that of $40 \mathrm{~N}$ applied in the present study. Another more likely reason could be the different method of force application to the ACL. While we used an isolated direct impact by means of a customized impactor, Friemert et al. achieved the mechanical stimulus by transferring a tensile force by a dropped weight that was connected to a cable pull via a pulley that led to the ACL. Krogsgaard et al. ${ }^{13}$ reported hamstring MLR values of 60 to 120 milliseconds after ACL or PCL stimulation. While the values in the present study were slightly lower than those of Krogsgaard et al., they were still in the same range with 79 milliseconds for the connection between the hamstring muscles and the ACL and 55 milliseconds (range 38-85 milliseconds) for the hamstring-PCL connection. The antagonistic function of the ACL and PCL is reflected in the different activation patterns of the ventral and dorsal thigh muscles. Following ACL stimulation, there was first a change in potential of the quadriceps muscles followed by a change in potential of the hamstrings. The reverse activation pattern can be clearly observed in our quantitative (Fig 4) and qualitative (Fig 5) "neuromapping" after PCL stimulation. The latency times reported by Beard et al. ${ }^{18}$ are within

Table 2. Tensile Forces, in N, of the Pretests

\begin{tabular}{lcccc}
\hline Structure & $\mathrm{F}_{\text {toe, }} \mathrm{N}$ & $\pm \mathrm{SD}, \mathrm{N}$ & $\mathrm{F}_{\max }, \mathrm{N}$ & $\pm \mathrm{SD}, \mathrm{N}$ \\
\hline $\mathrm{ACL}$ & 232.8 & 48.3 & $>500$ & - \\
PCL & 232.3 & 35.2 & $>500$ & - \\
LM & 163.8 & 130.7 & 294.0 & 167.9 \\
MM & 146.3 & 122.5 & 332.0 & 60.4 \\
HFP & 16.0 & 11.4 & 26.0 & 12.3 \\
PM & 14.8 & 9.7 & 47.3 & 17.8 \\
\hline
\end{tabular}

NOTE. The transition force $\left(\mathrm{F}_{\text {toe }}\right)$ is determined at the transition from the toe to the linear region of the force-elongation curves and the rupture force $\left(\mathrm{F}_{\max }\right)$ represents the maximum measured force. The ACL and PCL did not fail at the maximum applied load of $500 \mathrm{~N}$. ACL, anterior cruciate ligament; HFP, Hoffa's fat pad; LM, lateral meniscus; MM, medial meniscus; PCL, posterior cruciate ligament; PM, plica mediopatellaris; SD, standard deviation. 


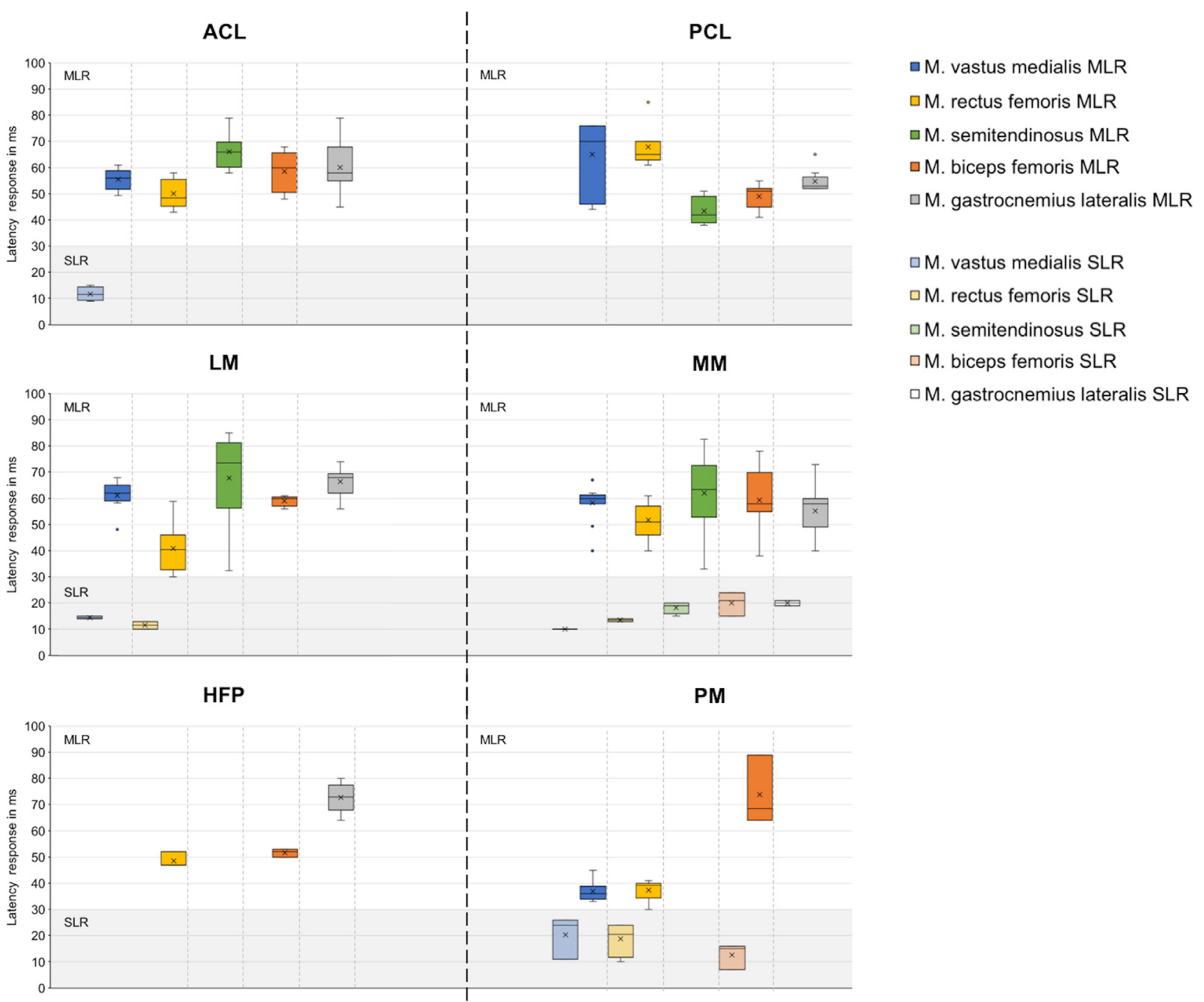

Fig 4. Mean (x), median, minimum, maximum, and 50\% values for the SLR and MLR, in milliseconds, of 5 knee-spanning muscles (M. vastus medialis, M. rectus femoris, M. semitendinosus, M. biceps femoris, and M. gastrocnemius lateralis) after isolated mechanical stimulation of the ACL $(n=15$ measurements), PCL $(n=12), L M(n=21), M M(n=24)$, HFP ( $n=18)$, and PM $(n=18)$. The gray area indicates the time range of the SLR $(\mathrm{t}<30 \mathrm{~ms})$ and the white area indicates the time range of the MLR ( $\mathrm{t} \geq 30 \mathrm{~ms}$ ) of the muscles. (ACL, anterior cruciate ligament; HFP, Hoffa's fat pad; LM, lateral meniscus; MLR, medium latency response; MM, medial meniscus; PCL, posterior cruciate ligament; PM, plica mediopatellaris; SLR, short latency response.)

our measurement range. However, it can be assumed that during a ventral tibial translation, the ACL was not stimulated in isolation. Therefore, the results are difficult to compare. Significantly longer MLR values after electrical stimulation were published by DyhrePoulsen and Krogsgaard $^{8}$ and Fischer-Rasmussen et al. ${ }^{9}$ However, it remains unclear which receptors were electrically stimulated, although a stimulation via group II and III fibers of the mechanoreceptors is suspected. Nevertheless, it should be noted that pain stimuli are transmitted via group III fibers. ${ }^{29}$ As already described in 1967 by Freeman and Wyke, ${ }^{34}$ the ACL contains Golgi tendon organs, which have a conduction velocity in the axon of $75 \mathrm{~m} / \mathrm{s}$. The distance in a human of $185 \mathrm{~cm}$ from the knee joint to the lumbar connection height L3/4 and back to the middle of the thigh is approximately $120 \mathrm{~cm}$. This leads to an arithmetical neural runtime of 16 milliseconds. Adding the multisynaptic connection with a delay of 1 millisecond per synapse and 3 milliseconds for the motor end plate, the reflex times measured in the present study are realistic. It further explains the MLR differences between the thigh and lower leg muscles with longer latencies of the M. gastrocnemius lateralis.

Friemert et al. ${ }^{35}$ also determined mean values of 40 milliseconds for the MLR of menisci after ventral tibial translation. It can be assumed that not only a mechanical stimulus of the menisci but also of the ACL 


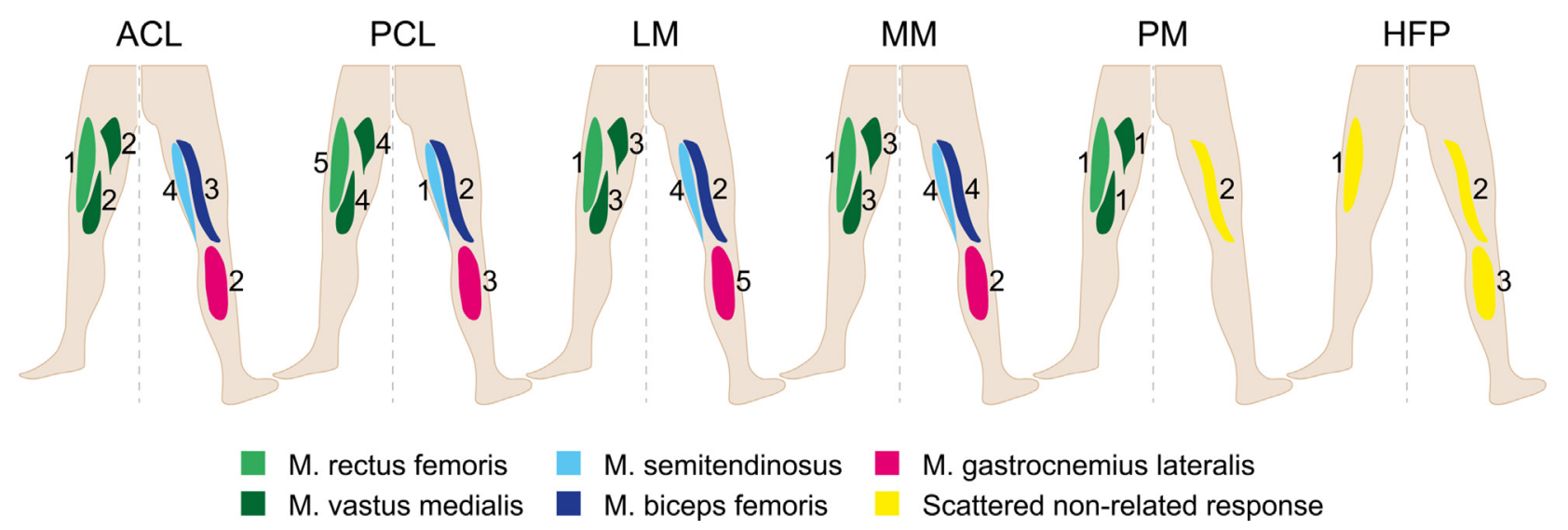

Fig 5. "MLR neuromapping": Anatomical related representation of the mean medium MLR patterns after isolated stimulation of the ACL, PCL, LM, MM, HFP, and PM. The different colors indicate the activation of the respective muscle except yellow, which indicates a scattered non-related response. The numbers indicate the activation sequence of the muscles. (ACL, anterior cruciate ligament; HFP, Hoffa's fat pad; LM, lateral meniscus; MLR, medium latency response; MM, medial meniscus; PCL, posterior cruciate ligament; PM, plica mediopatellaris.)

occurred during the translation maneuver. Therefore, a direct comparison of Friemert et al.'s results with the results of the present study is rather difficult.

Information about the knee joint capsule is only related to the histologic description of receptors. ${ }^{36}$ No neuromuscular, SLR, or MLR values have been reported to date. However, our findings indicated the shortest SLR values after the isolated mechanical stimulation of the PM. For the first time, we were able to perform an intraoperative detection of the reflex arc between the knee joint capsule and the thigh muscles, indicating latency times $<40$ milliseconds in the $\mathrm{M}$. vastus medialis. Further individual SLR changes occurred in the $M$. rectus femoris and $M$. biceps femoris. These findings indicate that the joint capsule, in addition to its function as a passive stabilizer, plays an important role in the neuromuscular connectivity. Being the anatomically most superficial component of the capsular ligament structures, it surrounds the entire knee joint and changes its tension state with very little movement. Therefore, we assume that the short latency period until the potential change of the musculature can be considered an early protective mechanism to compensate for further stretching of the other internal knee joint structures. Therefore, care should be taken during arthroscopic interventions to ensure minimal compromising of the joint capsule.

Regarding the HFP, individual reflex responses were recorded without reproducibility. Interindividual polymorphisms of the neurohistologic structure of the knee joint capsule with variable density or complete absence of receptors could be an explanation for the differences between the patients. Fischer-Rasmussen et al. ${ }^{9}$ used an electrical stimulus of the HFP and found no electromyography potential change of the thigh or lower leg muscles.
The results of the present study help to improve the understanding of the neuromuscular relationship of the thigh and shank muscles with the capsuloligamentous knee joint structures. It can be concluded that the better we understand the neuromuscular regulation of the knee joint, the better we are able to understand both the surgical and physiotherapeutic treatment of capsuloligamentous knee joint structures. ${ }^{37}$ After a knee joint injury, it is important to restore both the mechanical and proprioceptive deficits of the capsuloligamentous structures of the patient. To prevent long-term consequences, including the development of early osteoarthritis, the neuromapping that was introduced in the present study might indicate strategies to optimize a combined and therefore, more efficient, treatment of the proprioceptive deficit. A postoperative treatment could be implemented in the form of neuromuscular training within the framework of physiotherapeutic exercises. ${ }^{38}$ One possible option for neuromuscular training in the immediate postoperative phase is the use of controlled active motion devices for patients after ACL or PCL reconstruction ${ }^{39}$ or neuromuscular electrical stimulation for neuromuscular

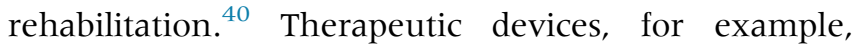
Aerostep, stepper, trampoline, bodyblade and the Posturomed, are useful in the rehabilitation process to train specific neuromuscular deficits. However, based on the results of the present study such exercises should not only be performed after ACL injuries, ${ }^{41}$ but also for postoperative rehabilitation after PCL, meniscus or joint capsule injuries.

\section{Limitations}

One of the main limitations of the study is the low number of patients involved. Because of the different indications for the arthroscopic interventions, it was not 
Table 3. Literature Overview of Medium Latency Response Values (MLR) in milliseconds, Resulting From Mechanical or Electrical Stimulation of Different Capsuloligamentous Structures on the Lower Leg Muscles

\begin{tabular}{|c|c|c|}
\hline Structure & Author & $\begin{array}{c}\text { Target Muscle and Related MLR } \\
\text { Range }\end{array}$ \\
\hline$\overline{\mathrm{ACL}}$ & $\begin{array}{l}\text { Friemert et al., } \\
2005^{29}\end{array}$ & Hamstrings: $44 \pm 4 \mathrm{~ms}$ \\
\hline ACL & $\begin{array}{l}\text { Friemert et al., } \\
2005^{28}\end{array}$ & Hamstrings: $40-50 \mathrm{~ms}$ \\
\hline ACL / PCL & $\begin{array}{l}\text { Krogsgaard } \\
\quad \text { et al., } 2002^{13}\end{array}$ & Hamstrings: $60-120 \mathrm{~ms}$ \\
\hline ACL & $\begin{array}{l}\text { Beard et al., } \\
1994^{7}\end{array}$ & Hamstrings: 50-99 ms \\
\hline ACL & $\begin{array}{l}\text { Dyhre-Poulsen } \\
\text { and } \\
\text { Krogsgaard. } \\
2000^{8}\end{array}$ & M. semitendinosus: $95 \pm 35 \mathrm{~ms}^{*}$ \\
\hline ACL & $\begin{array}{l}\text { Tsuda et al., } \\
2001^{34}\end{array}$ & Hamstrings: $50-80 \mathrm{~ms}^{*}$ \\
\hline PCL & $\begin{array}{l}\text { Fischer- } \\
\quad \text { Rasmussen } \\
\text { et al., } 2002^{9}\end{array}$ & $\begin{array}{l}\text { M. quadriceps: } 78-148 \mathrm{~ms}^{*} \\
\text { Hamstrings: } 88-110-\mathrm{ms}^{*} \\
\text { M. gastrocnemius: } 189-258 \mathrm{~ms}^{*}\end{array}$ \\
\hline Menisci & $\begin{array}{l}\text { Friemert et al., } \\
2007^{35}\end{array}$ & Hamstrings: $39 \pm 3 \mathrm{~ms}$ \\
\hline ACL & Present study & $\begin{array}{l}\text { M. vastus medialis: } 49-61 \mathrm{~ms} \\
\text { M. rectus femoris: } 43-58 \mathrm{~ms} \\
\text { M. semitendinosus: } 58-79 \mathrm{~ms} \\
\text { M. biceps femoris: } 48-68 \mathrm{~ms} \\
\text { M. gastrocnemius lateralis: } 45- \\
79 \mathrm{~ms}\end{array}$ \\
\hline PCL & & $\begin{array}{l}\text { M. vastus medialis: } 44-76 \mathrm{~ms} \\
\text { M. rectus femoris: } 61-85 \mathrm{~ms} \\
\text { M. semitendinosus: } 38-51 \mathrm{~ms} \\
\text { M. biceps femoris: } 41-55 \mathrm{~ms} \\
\text { M. gastrocnemius lateralis: } 52- \\
65 \mathrm{~ms}\end{array}$ \\
\hline LM & & $\begin{array}{l}\text { M. vastus medialis: } 41-68 \mathrm{~ms} \\
\text { M. rectus femoris: } 56-62 \mathrm{~ms} \\
\text { M. semitendinosus: } 32-85 \mathrm{~ms} \\
\text { M. biceps femoris: } 56-62 \mathrm{~ms} \\
\text { M. gastrocnemius lateralis: } 56- \\
74 \mathrm{~ms}\end{array}$ \\
\hline MM & & $\begin{array}{l}\text { M. vastus medialis: } 49-67 \mathrm{~ms} \\
\text { M. rectus femoris: } 40-61 \mathrm{~ms} \\
\text { M. semitendinosus: } 33-83 \mathrm{~ms} \\
\text { M. biceps femoris: } 55-78 \mathrm{~ms} \\
\text { M. gastrocnemius lateralis: } 40- \\
73 \mathrm{~ms}\end{array}$ \\
\hline PM & & $\begin{array}{l}\text { M. vastus medialis: } 33-45 \mathrm{~ms} \\
\text { M. rectus femoris: } 30-41 \mathrm{~ms} \\
\text { M. biceps femoris: } 64-89 \mathrm{~ms}\end{array}$ \\
\hline
\end{tabular}

ACL, anterior cruciate ligament; LM, lateral meniscus; MM, medial meniscus; PCL, posterior cruciate ligament; PM, plica mediopatelaris. ${ }^{*}$ MLR response after electrical stimulation-all other values result after mechanical stimulation.

possible to investigate all structures in each patient (ACL: $\mathrm{n}=5$; PCL: $\mathrm{n}=4$; LM: $\mathrm{n}=7$; MM: $\mathrm{n}=8$; PM: $\mathrm{n}=6$; HFP: $\mathrm{n}=6$ ). Therefore, the number of measurements per structure obtained in the present study is too low to allow an adequate statistical evaluation.
Further, it remains to be examined what role individual reflex responses play after isolated mechanical stimulation of the HFP. A more reproducible HFP reflex outcome may be achievable with more patients and higher minimum values of the mechanical stimulation of the HFP. The latter can be determined, for example, using human specimens during pretests.

\section{Conclusions}

Each stimulated structure displayed an individual MLR response, which allowed us to create neuromapping combining the anatomical and quantitative representations of the individual muscular activation patterns after isolated mechanical stimulation of the capsuloligamentous knee joint structures, corroborating our hypothesis.

\section{Acknowledgments}

We thank Dr. Schwarz, Dr. Thürigen, Dr. Herr, Dr. Bülow, and Dr. Riesner from the Department of Trauma and Orthopedic Surgery of the Hospital of the Federal Armed Forces, Ulm, for their support during the in vivo measurements. Furthermore, we thank Patrizia Horny from the Institute of Orthopedic Research and Biomechanics Ulm for her design support and Herbert Schmitt for his efforts and support in the mechanical design of the impactor device.

\section{References}

1. Majewski M, Susanne H, Klaus S. Epidemiology of athletic knee injuries: A 10-year study. Knee 2006;13: 184-188.

2. Fischer JF, Leyvraz PF, Bally A. A dynamic analysis of knee ligament injuries in alpine skiing. Acta Orthop Belg 1994;60:194-203.

3. Upmeier $\mathrm{H}$, Bruggenjurgen B, Weiler A, Flamme C, Laprell H, Willich SN. Follow-up costs up to 5 years after conventional treatments in patients with cartilage lesions of the knee. Knee Surg Sports Traumatol Arthrosc 2007;15: 249-257.

4. Shimokochi Y, Shultz SJ. Mechanisms of noncontact anterior cruciate ligament injury. J Athl Train 2008;43: 396-408.

5. Biedert RM, Zwick EB. Ligament-muscle reflex arc after anterior cruciate ligament reconstruction: Electromyographic evaluation. Arch Orthop Trauma Surg 1998;118: 81-84.

6. Beard DJ, Dodd CA, Trundle HR, Simpson AH. Proprioception enhancement for anterior cruciate ligament deficiency. A prospective randomised trial of two physiotherapy regimes. J Bone Joint Surg Br 1994;76: 654-659.

7. Beard DJ, Kyberd PJ, Dodd CA, Simpson AH, O'Connor JJ. Proprioception in the knee. J Bone Joint Surg Br 1994;76:992-993. 
8. Dyhre-Poulsen P, Krogsgaard MR. Muscular reflexes elicited by electrical stimulation of the anterior cruciate ligament in humans. J Appl Physiol (1985) 2000;89:2191-2195.

9. Fischer-Rasmussen T, Krogsgaard MR, Jensen DB, DyhrePoulsen P. Muscle reflexes during gait elicited by electrical stimulation of the posterior cruciate ligament in humans. J Orthop Res 2002;20:433-438.

10. Hogervorst T, Brand RA. Mechanoreceptors in joint function. J Bone Joint Surg Am 1998;80:1365-1378.

11. Jerosch J, Prymka M. Proprioception and joint stability. Knee Surg Sports Traumatol Arthrosc 1996;4:171-179.

12. Johansson H. Role of knee ligaments in proprioception and regulation of muscle stiffness. J Electromyogr Kinesiol 1991;1:158-179.

13. Krogsgaard MR, Dyhre-Poulsen P, Fischer-Rasmussen T. Cruciate ligament reflexes. J Electromyogr Kinesiol 2002;12: 177-182.

14. Solomonow M, Baratta R, Zhou BH, et al. The synergistic action of the anterior cruciate ligament and thigh muscles in maintaining joint stability. Am J Sports Med 1987; 15:207-213.

15. Chmielewski TL, Hurd WJ, Snyder-Mackler L. Elucidation of a potentially destabilizing control strategy in ACL deficient non-copers. J Electromyogr Kinesiol 2005;15:83-92.

16. Hurd WJ, Axe MJ, Snyder-Mackler L. Influence of age, gender, and injury mechanism on the development of dynamic knee stability after acute ACL rupture. J Orthop Sports Phys Ther 2008;38:36-41.

17. Kaplan Y. Identifying individuals with an anterior cruciate ligament-deficient knee as copers and noncopers: A narrative literature review. J Orthop Sports Phys Ther $2011 ; 41: 758-766$.

18. Beard DJ, Kyberd PJ, O'Connor JJ, Fergusson CM, Dodd CA. Reflex hamstring contraction latency in anterior cruciate ligament deficiency. J Orthop Res 1994;12:219-228.

19. Fujita I, Nishikawa T, Kambic HE, Andrish JT, Grabiner MD. Characterization of hamstring reflexes during anterior cruciate ligament disruption: In vivo results from a goat model. J Orthop Res 2000;18:183-189.

20. Miyatsu M, Atsuta Y, Watakabe M. The physiology of mechanoreceptors in the anterior cruciate ligament. An experimental study in decerebrate-spinalised animals. J Bone Joint Surg Br 1993;75:653-657.

21. Raunest J, Sager M, Burgener E. Proprioceptive mechanisms in the cruciate ligaments: An electromyographic study on reflex activity in the thigh muscles. J Trauma 1996;41:488-493.

22. Kennedy JC, Alexander IJ, Hayes KC. Nerve supply of the human knee and its functional importance. Am J Sports Med 1982;10:329-335.

23. Zimny ML, Wink CS. Neuroreceptors in the tissues of the knee joint. J Electromyogr Kinesiol 1991;1:148-157.

24. Barrett DS, Cobb AG, Bentley G. Joint proprioception in normal, osteoarthritic and replaced knees. J Bone Joint Surg Br 1991;73:53-56.

25. Lattanzio PJ, Petrella RJ. Knee proprioception: A review of mechanisms, measurements, and implications of muscular fatigue. Orthopedics 1998;21:463-470. discussion 470-461; passim.

26. Jennings AG, Seedhom BB. Proprioception in the knee and reflex hamstring contraction latency. J Bone Joint Surg Br 1994;76:491-494.
27. Mine T, Kimura M, Sakka A, Kawai S. Innervation of nociceptors in the menisci of the knee joint: an immunohistochemical study. Arch Orthop Trauma Surg 2000;120:201-204.

28. Friemert B, Bumann-Melnyk M, Faist M, Schwarz W, Gerngross H, Claes L. Differentiation of hamstring short latency versus medium latency responses after tibia translation. Exp Brain Res 2005;160:1-9.

29. Friemert B, Faist M, Spengler C, Gerngross H, Claes L, Melnyk M. Intraoperative direct mechanical stimulation of the anterior cruciate ligament elicits short- and medium-latency hamstring reflexes. J Neurophysiol 2005;94:3996-4001.

30. Kerz T, Hennes HJ, Feve A, Decq P, Filipetti P, Duvaldestin P. Effects of propofol on H-reflex in humans. Anesthesiology 2001;94:32-37.

31. Hermens HJ, Freriks B, Disselhorst-Klug C, Rau G. Development of recommendations for SEMG sensors and sensor placement procedures. J Electromyogr Kinesiol 2000;10:361-374.

32. Woo SL, Debski RE, Withrow JD, Janaushek MA. Biomechanics of knee ligaments. Am J Sports Med 1999;27:533-543.

33. Faul F, Erdfelder E, Lang AG, Buchner AG. *Power 3: A flexible statistical power analysis program for the social, behavioral, and biomedical sciences. Behav Res Methods 2007;39:175-191.

34. Freeman MA, Wyke B. Articular reflexes at the ankle joint: an electromyographic study of normal and abnormal influences of ankle-joint mechanoreceptors upon reflex activity in the leg muscles. Br J Surg 1967;54: 990-1001.

35. Friemert B, Wiemer B, Claes L, Melnyk M. The influence of meniscal lesions on reflex activity in the hamstring muscles. Knee Surg Sports Traumatol Arthrosc 2007;1 5:1198-1203.

36. Freeman MA, Wyke B. The innervation of the knee joint. An anatomical and histological study in the cat. J Anat 1967; 101:505-532.

37. van Melick N, van Cingel RE, Brooijmans F, et al. Evidence-based clinical practice update: practice guidelines for anterior cruciate ligament rehabilitation based on a systematic review and multidisciplinary consensus. $\mathrm{Br} J$ Sports Med 2016;50:1506-1515.

38. Bregenhof B, Jorgensen U, Aagaard P, et al. The effect of targeted exercise on knee-muscle function in patients with persistent hamstring deficiency following ACL reconstruction-study protocol for a randomized controlled trial. Trials 2018;19:75.

39. von Lübken F, Friemert B, Schmidt R, Jouini C, Gerngross H. The influence of a controlled active motion splint on proprioception after anterior cruciate ligament plasty. A prospective randomized study. Der Unfallchirurg 2006;109:22-29 [in German].

40. Maffiuletti NA, Gondin J, Place N, Stevens-Lapsley J, Vivodtzev I, Minetto MA. Clinical use of neuromuscular electrical stimulation for neuromuscular rehabilitation: What are we overlooking? Arch Phys Med Rehabil 2018;99:806-812.

41. Kaya D, Guney-Deniz H, Sayaca C, Calik M, Doral MN. Effects on lower extremity neuromuscular control exercises on knee proprioception, muscle strength, and functional level in patients with ACL reconstruction. Biomed Res Int 2019;2019:1694695. 